\title{
A New Routing Scheme Concerning Power-Saving in Mobile Ad-Hoc Networks
}

\author{
Jung-hee Ryu and Dong-Ho Cho \\ Department of Electrical Engineering and Computer Science \\ Korea Advanced Institute of Science and Technology (KAIST) \\ Taejon, Korea. PH: +82-42-869-8067, FAX: +82-42-867-0550 \\ e-mail: $\{r y u, d h c h o\} @ e e . k a i s t . a c . k r$
}

\begin{abstract}
In the mobile ad-hoc network, there can exist two categories of terminals, the battery-powered and the outlet-plugged terminals. In this paper, a new scheme for the power-saving of battery-powered terminals in mobile ad-hoc networks is proposed. Based on this scheme, the battery. powered terminals page the traffic with minimum power to the neares outlet-plugged terminal, and only the outlet-plugged terminals are used as the routing terminals. The simulation results show that the mean radius of the battery-powered terminal's paging area are much smaller than that of the other conventional schemes.
\end{abstract}

\section{INTRODUCTION}

Today, the technological innovation and the market explosion in the fields of networking and computing have stimulated much public interest and consumers demand even more advanced network services. Especially, the demands of local area network (LAN) for office or home environments are rapidly increasing, and the conventional fixed wired LAN environments are evolving to the wireless mobile networks. Recently, the mobile ad-hoc network is treated as the candidate of next generation wireless LAN environment. There exist no special routers or switches for communication in mobile ad-hoc networks, but all or some terminals take a role of those devices. Hence, dynamic configurations of terminals are possible in mobile ad-hoc networks, without any setting of devices. Because of this advantage, many researches and developments on mobile ad-hoc networks are in progress by various groups such as IETF MANET WG and Bluetooth SIG.

Because a mobile ad-hoc network is a multi-hop wireless network in which mobile hosts communicate over a shared and limited radio channel, a mobile ad-hoc network is characterized by the lack of a wired backbone or centralized entities. Thus, in ad-hoc networks, we need more sophisticated distributed algorithms to perform these functions of coordination[1]. Since all communications between all network elements are carried within limited resources, we have much constraint on the efficient network management for mobile ad-hoc networks; e.g. conservation of wireless spectrum and reduction of transmission power. Thus, for communication between two entities in ad-hoc network, we have to apply multi-hop routing to be relayed through intermediate nodes. In addition, since the network topology is changed rapidly in ad-hoc networks, it is very significant and difficult problem to find and maintain an optimized route. Therefore, ad-hoc routing algorithm must react quickly to topology changes.

The architecture of an ad-hoc network can be either flat or $h i$ - erarchical[1]. In a hierarchical network, the network elements are partitioned into several groups, called cluster. In each cluster, there is a cluster head, which is selected to manage all the other nodes within the cluster. The depth of the network can vary from a single tier to multiple tiers. In a flat network, all nodes have equal rank. After all, flat networks are equivalent to zero-tier hierarchical networks.

Hierarchical network architecture can accomplish the mobility management process more easily, but routing is often suboptimal because of the lack of direct connectivity between two different cluster heads. On the other hand, the most important advantage of the flat network is that there are multiple paths between source and destination. This allows congestion reduction and we can choose the best route to satisfy the specific requirements of a traffic attribute. While flat addressing may be less complicated and easier to use, there are doubts as to its scalability.

In the case of flat network, each source terminal pages the traffic to neighboring terminals, and these terminals page again until the destination terminal receives the traffic from the corresponding source terminal. The terminals which take a role of the router are named as routing terminal. The paging area of a terminal is related to the transmission power of itself, therefore a wider paging area means a higher transmission power. But in the applications of mobile ad-hoc networks such as office or home area networks, there exist two categories of terminals, battery-powered terminals and outlet-plugged terminals. The battery-powered terminal means the terminal that has only limited energy source, such as battery. The examples of terminals in this category are notebooks, PDAs, and remote controllers. These terminals have relatively high mobility and small size. On the contrary, the outlet-plugged terminal has stable and durable energy source. The examples of the mobile terminals are desktop computers, servers, and TVs. In the case of the batterypowered terminals, the energy for data transmission is limited and the power saving is one of very important feature. On the contrary, the power saving is not a major consideration for the outlet-plugged terminals, and the higher transmission power is required to make the paging area larger.

In this paper, we propose a new scheme for the power saving of battery-powered terminals in mobile ad-hoc networks considering above antithesis of two terminal types. In this proposed scheme, the battery-powered terminals page with mini- 
mum power level to transmit the traffic to the nearest outletplugged terminal, and the outlet-plugged terminal pages this traffic to destination terminal or routing terminal with maximum power level allowed to itself. And the battery-powered terminals are not used as the routing terminals, except the case there exist no outlet-plugged terminal to be used. In the case of the outlet-plugged terminals, the traffic is paged to destination terminals or routing terminals with maximum power level allowed to themselves to decrease hop numbers from the source to the destination terminal.

The performances of the proposed scheme and conventional scheme based on the constant paging power level are simulated using the Cadence BONeS DESIGNER. As performance measures, the mean radius of the battery-powered terminal's paging area, the call blocking probability and the call dropping probability are concerned. The numerical results show that the average consumed energy of the battery-powered terminals for paging and the call dropping probability are much lower than those in the conventional scheme, while the call blocking probability is higher.

Following this introduction, we describe the proposed scheme in Section II. In Section III, we describe the simulation environment, and in Section IV, we discuss numerical results. Finally, we make conclusions in Section V.

\section{Descriptions on the Proposed Routing SCHEME}

In the proposed scheme, the networks are flat and the cluster of a terminal is the same as the paging area of it. The source terminal that is battery-powered pages with minimum power level to transmit the traffic to the nearest outlet-plugged terminal, and that outlet-plugged terminal pages this traffic to destination terminal or routing terminal with maximum power level allowed to itself. In the case of the outlet-plugged terminals, the traffic is paged to destination terminals or routing terminals with maximum power level allowed to themselves to decrease hop numbers from the source to the destination terminal. In each case, if there exists no outlet-plugged terminal within the maximum paging area of the source or the routing terminal, the call is blocked.

As described above, the candidate terminals as routers in the proposed scheme are only outlet-plugged terminals. Like the conventional demand-driven schemes, when the source terminal does not find the target terminal in the cluster of itself, the routing path discovery scheme is started. But the difference between conventional schemes and the proposed scheme is that the paging signals to the terminals in the cluster are received by only outlet-plugged terminals. When the outlet-plugged terminal receives the paging signals, there exist two possible schemes to make routing path.

One is the table-driven approach. The updating rates of routing tables among outlet-plugged terminals are comparatively low, hence the prepared routing table can be used without paging to find the routing path. Each battery-powered terminals are registered in the memory of the outlet-plugged terminals when the battery-powered terminals enters into the cluster of the low mobility terminals, and if one of the outlet-plugged terminals receives the paging signals from the source terminal, then the outlet-plugged terminal multicasts the terminal finding signal to the all outlet-plugged terminals in the prepared routing table. Then, the outlet-plugged terminals seek the target terminal based on memory contents. With this approach, the routing time can be shortened but the capability of each outlet-plugged terminals to manage memory contents are also needed. The other approach is demand-driven. Each outlet-plugged terminal receiving the paging signals for routing finds the target terminal in its cluster, and if the finding fails then it forwards the routing signal to the outlet-plugged terminals in its cluster. This approach is more simple than the first approach, but the number of paging to find route is larger than the case of the first approach. A detailed process of the proposed routing scheme with demand-driven approach is shown in Figure 1.

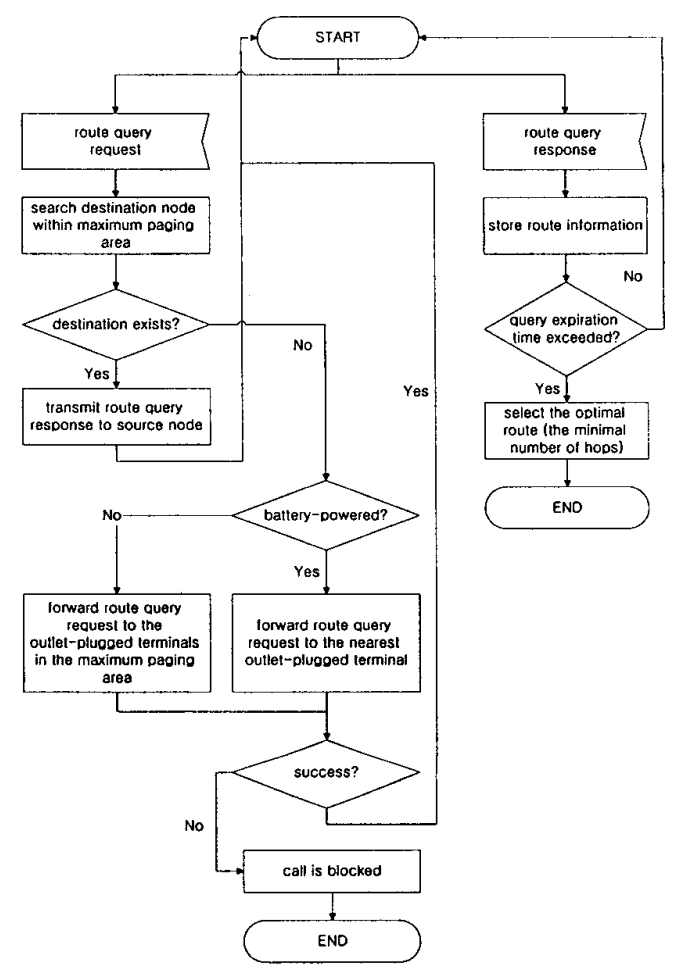

Fig. 1. A flow chart for the proposed routing scheme

In Figure 2, an example of the proposed routing algorithm is shown. The solid lines in this figure represent the routing path by the proposed scheme $(s-A-B-C-D-t)$, and the dotted lines represent the routing path by the conventional scheme without the concerning of the power-saving $(s-B-a-t)$. Even though the hops in the routing path is smaller in the case of the conventional scheme, the paging area of the battery-powered terminal $\mathrm{s}$ is much smaller than those in the conventional case.

\section{Simulation ENVIRONMENTS}

In this section, we describe about the simulation environments for the performance evaluation of the proposed scheme. We use the Cadence BONeS DESIGNER network simulator, which is an event driven simulation package. 


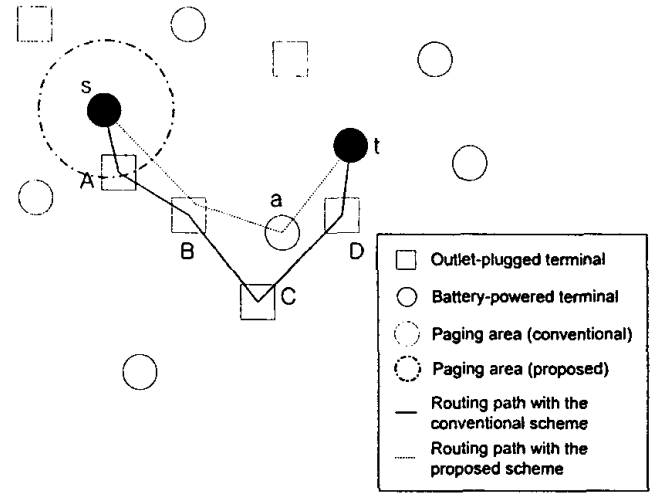

Fig. 2. An example of the routing path with the proposed routing scheme

Our simulated network consists of 12,20 and 28 mobile nodes, whose initial positions are chosen from a uniform random distribution over an area of $10 \mathrm{~m}$ by $10 \mathrm{~m}$. All nodes move at a constant speed, $v$, with an initial direction, $\theta$, which is uniformly distributed between 0 and $2 \pi$. When a node reaches the edge of the simulation region, it is reflected back into the coverage area, by setting its direction to $-\theta$ (horizontal edges) or $\pi-\theta$ (vertical edges). The magnitude of the velocity is not altered.

We assume that the outlet-plugged terminals have relatively low mobility, and the battery-powered terminals have relatively high mobility. And we assume that the routing path finding approach is demand-driven. The terminals in the conventional scheme and the outlet-plugged terminals are blocked when the routing path finding is failed within 4 hops. In the case of the battery-powered terminal, the nearest outlet-plugged terminal operates as the source terminal for the routing path finding. In other words, the nearest outlet-plugged terminal of the source battery powered terminal tries to establish the routing path to the target terminal within 4 hops. Hence, the maximum allowed number of hops for the battery-powered terminal is 5 .

The simulation of the proposed scheme is based on the assumption that the network topology remains fixed during a route discovery. Also, we assume that there is no MAC layer channel contention. This assumption prevents the delay measurements from being biased by the delays associated with any particular MAC collision avoidance scheme. In addition, we assume that any packet can be received in error-free condition, within a radius of $r_{\max }$ from the transmitter, but could be lost beyond $r_{\max }$. Here, $r_{\max }$ is the maximum radius of the paging area, i.e. cluster area for each node.

In the conventional routing scheme, all nodes can be the routing terminals, and all source terminals find destination terminals or routing terminals within their maximum paging area. and speeds of all terminals are defined by the same uniform distribution.

The simulation parameters mentioned above are shown in Table I.
SIMULATION PARAMETERS

\begin{tabular}{|c|c|}
\hline Parameter (Symbol) & Value \\
\hline $\begin{array}{c}\text { Network coverage area } \\
\left(L_{x} \times L_{y}\right)\end{array}$ & $10 \mathrm{~m} \times 10 \mathrm{~m}$ \\
\hline Number of nodes $(n)$ & $12,20,28$ \\
\hline Speed of battery-powered & Uniform distribution \\
terminal $\left(v_{B T}\right)$ & $(1-2 \mathrm{~m} / \mathrm{s})$ \\
\hline $\begin{array}{c}\text { Speed of outlet-plugged } \\
\text { terminal }\left(v_{O T}\right)\end{array}$ & $\begin{array}{c}\text { Uniform distribution } \\
(0-1 \mathrm{~m} / \mathrm{s})\end{array}$ \\
\hline Speed of terminals & Uniform distribution \\
in the conventional case $\left(v_{c}\right)$ & $(0-2 \mathrm{~m} / \mathrm{s})$ \\
\hline Initial direction of node $(\theta)$ & Uniform distribution \\
& $(0-2 \pi)$ \\
\hline Maximum paging area & $8 \mathrm{~m}$ \\
radius $\left(r_{\text {max }}\right)$ & $25,50,75 \%$ \\
\hline Percentage of battery-powered \\
terminals in all nodes $\left(P_{B T}\right)$
\end{tabular}

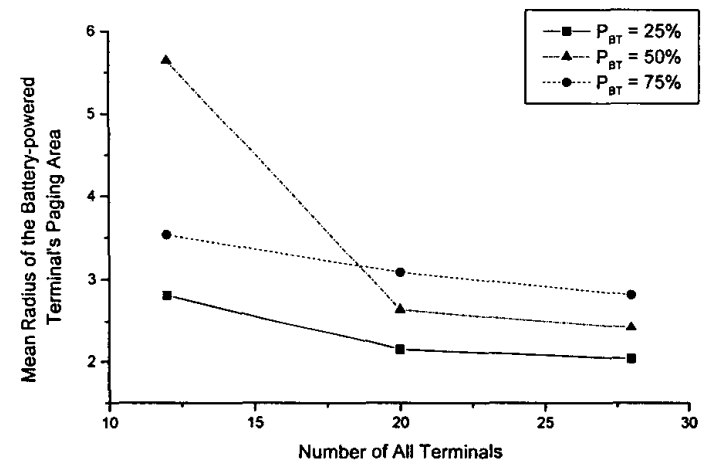

Fig. 3. The mean radius of the battery-powered terminal's paging area vs. number of all terminals

\section{NumERICAL RESULTS}

In this simulation, we choose the mean radius of the batterypowered terminal's paging area, the call blocking probability and the call dropping probability as performance measures. The mean call blocking probability is defined as the probability that the new call blocking occurs when neither the destination terminal nor the routing terminal exists in the maximum paging area of the source terminal and the routing terminals between the source and the destination terminal, and the mean call dropping probability is defined as the probability that the call dropping occurs because of the disconnection between two routing terminals in the routing path. The numerical results related with these measures are shown in Figure 3, 4 and 5.

First, we can see that the mean radiuses of the batterypowered terminal's paging area in the proposed scheme are $30-70 \%$ smaller than the radius of the paging area in the conventional scheme, $r_{\max }, 8 \mathrm{~m}$ as shown in Figure 3 . The mean radius of the battery-powered terminal's paging area is 


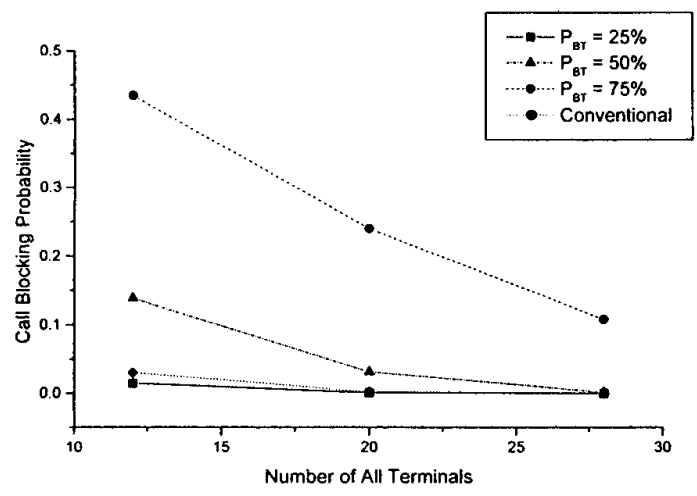

Fig. 4. Call blocking probability vs. number of all terminals

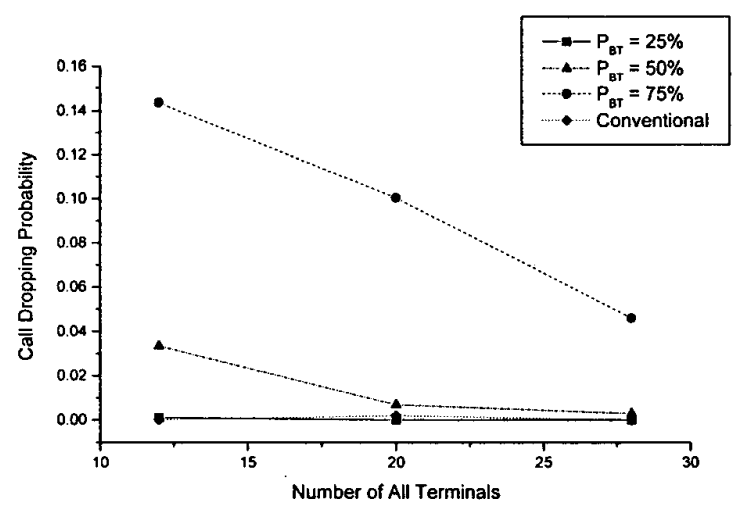

Fig. 5. Call dropping probability vs. number of all terminals

decreased with the increasing of the number of mobile terminals, because the probability that outlet-plugged terminals exist at nearer position is higher. When the percentage of batterypowered terminals in all nodes, $P_{B T}$, is increased, the number of outlet-plugged terminals is decreased, hence the mean radius of the battery-powered terminal's paging area is increased. But, when the number of terminals are relatively small, in the case of lower $P_{B T}$, the number of outlet-plugged terminals are increased hardly to make the new call successful. Then, the mean radius of the non-blocked battery-powered terminal's paging area can be relatively decreased. The case with $P_{B T}=75 \%$ and $N=10$ is the example of this phenomenon.

As shown in Figure 4, the call blocking probabilities in the proposed scheme when $P_{B T}$ is 50 or $75 \%$ are higher than that in the conventional scheme, because outlet-plugged terminals only can be the routing terminals in the proposed scheme. It is more difficult to set the routing path successfully based on this characteristics. The call blocking probability with $P_{B T}=25 \%$ is slightly lower than the conventional case, because the batteryplugged terminal in the proposed scheme can try maximum 5 hops to find a routing path. The maximum number of hops limited in the conventional scheme is 4 as mentioned in the previous section. The call blocking probability is decreased with the increasing of the number of mobile terminals, because the number of routing terminals are also increased. With the increasing of $P_{B T}$, the number of outlet-plugged terminals that have the roles of the routing terminal is decreased, and the call blocking probability is increased.

In the case of the call dropping probabilities, numerical results are similar to the call blocking probabilities. As described in the above paragraph, the relatively small number of routing terminals in the proposed scheme affects these results. The similarity of numerical results between the conventional case and the proposed case with $P_{B T}=25 \%$ is also explained by the difference of the maximum number of hops described previously.

\section{CONCLUSIONS}

In this paper, we proposed the new routing scheme concerning power-saving of terminals in mobile ad-hoc networks to overcome the power dissipation problem of the battery-powered terminals. In the proposed scheme, the source terminal that is battery-powered pages with minimum power level to transmit the traffic to the nearest outlet-plugged terminal, and only the outlet-plugged terminals can be the routing terminal. Because the outlet-plugged terminal has low-mobility, both of the tabledriven and the demand-driven approaches can be used to find the routing paths in the proposed scheme. With the proposed scheme, the radius of the battery-powered terminal's paging area is much smaller than that in the conventional scheme. Hence, the power dissipation of battery-powered terminal can be decreased efficiently. The numerical results show that the average consumed energy of the battery-powered terminals for paging and the call dropping probability are somewhat lower than those in the conventional scheme, while the call blocking probability increases slightly.

As a further work, the scheme that uses the battery-powered terminal as a routing terminal will be studied. With the currently proposed scheme, the call blocking probability is relatively higher than that in the conventional scheme, because only the outlet-plugged terminal can be a routing terminal. If the battery-powered terminal can have the role of a routing terminal when the routing for new call initiation based on only the outletplugged terminals fails, the call blocking probability could be reduced effectively.

\section{REFERENCES}

[1] Zygmunt J. Hass and Siamak Tabrizi, "On Some Challenges and Design Choices in Ad-Hoc Communications", Proc. of MlLCOM'98, October 1998

[2] Elizabeth M. Royer and Chai-Keong Toh, "A Review of Current Routing Protocols for Ad-Hoc Mobile Wireless Networks", IEEE Personal Communications, April 1999

[3] Charles. E. Perkins and P. Bhagwat, "Highly Dynamic DestinationSequenced Distance-Vector Routing (DSDV) for Mobile Computers", ACM SIGCOMM, vol. 24, no. 4, October 1994

[4] J. Broch, D. B. Johnson and D. A. Maltz, The Dynamic Source Routing Protocol for Mobile Ad-Hoc Networks, IETF Internet Draft, December 1998

[5] Charles. E. Perkins and Elizabeth. M. Royer, Ad-Hoc On Demand Dis. tance Vector (AODV) Routing, IETF Intemet Draft, November 1998

[6] Zygmunt J. Hass and M. R. Pearlman, The Zone Routing Protocol (ZRP) for Ad-Hoc Networks, IETF Internet Draft, December 1997 